\title{
A QUANTITATIVE STUDY OF REGENERATION BY INDUCTIVE FEED BACK.
}

\author{
By C. B. Jolliffe and Miss J. A. Rodman.
}

\begin{abstract}
.
The use of inductive coupling between the tuned grid circuit and plate circuit of a radio-receiving circuit network employing an electron tube has been extensively used for the amplification of received signals since its discovery by E. H. Armstrong. Using simple alternating-current theory this paper shows that the amplification produced by this type of regeneration in an amplifying circuit network can be considered as being caused by a reduction in the resistance of the tuned circuit. The current amplification produced in the tuned circuit for any value of impressed radiofrequency signal is

where

$$
\frac{I_{1}}{I_{0}}=\frac{R_{1}}{\left(R_{1}-a\right)-\frac{\mu M}{C_{1} R_{2}}}
$$

$R_{1}$ is the resistance of the tuned circuit,

$M$ is the mutual inductance which produces the feed back,

$\mu$ the voltage amplification factor of the tube,

$R_{2}$ the total resistance of the plate circuit,

$C_{1}$ the capacity in the tuned circuit across which the tube is connected,

$a$ the reduction in resistance produced by the capacitive feed back through the tube.

The voltage of the grid was assumed to be negative at all times.

The above equation was completely confirmed by experiment. The current in the tuned circuit with and without regeneration was measured by means of an electrontube voltmeter which measured the drop of potential across the capacity $C_{1}$ in the tuned circuit. The reduction in resistance $a$ caused by capacitive feed back was obtained experimentally by the resistance variation method.
\end{abstract}

\section{CONTENTS.}

Page.

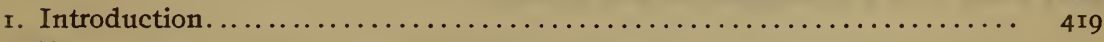

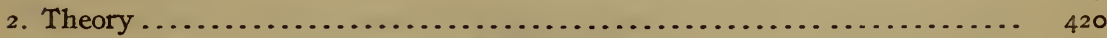

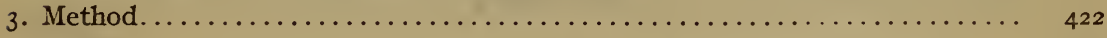

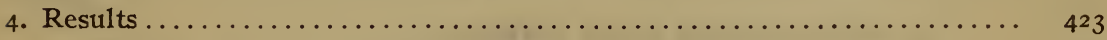

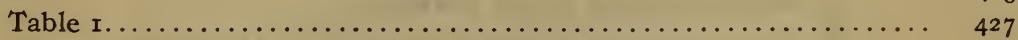

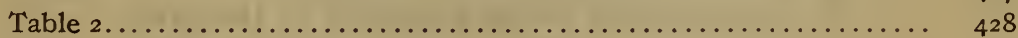

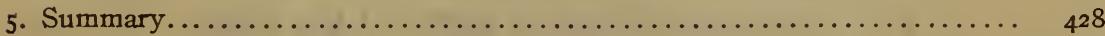

\section{INTRODUCTION.}

The electron-tube circuit network by which received radio signals may be strengthened materially by coupling the grid and plate circuits of the tube were first described by E. H. Armstrong. ${ }^{1}$

1 Proc. I. R. E., 3, p. 215; 1915. 
The principal methods used for obtaining this regeneration are (I) capacitive coupling using usually the capacity of the tube itself, (2) inductive coupling where a coil in the plate circuit and a coil in the tuned grid circuit are inductively coupled. A short time after the discovery of this phenomenon the idea was advanced that regeneration in effect introduced a negative resistance in a tuned circuit. This idea has been variously interpreted and at times questioned. ${ }^{2}$

Miller ${ }^{3}$ studied theoretically and experimentally the case of capacitive coupling, and showed that because of the capacities between the elements of an electron tube the input impedance of the tube depends on the nature of the load in the plate circuit. If this load in the plate circuit is inductive, it was shown that the input impedance can be characterized as a negative resistance, in which case the resistance of the tuned circuit is apparently reduced. The equations for calculating the amount of reduction of the resistance are given. ${ }^{4}$

Very little quantitative data are available concerning regeneration which is obtained by inductive coupling between the output and input circuits of a tube, and this work is to show by experiment that the results of regeneration by inductive feed back may be calculated from simple alternating-current theory.

\section{THEORY.}

The circuit network as ordinarily used is shown in Figure $r$. $L$ is the total inductance in the tuned circuit, $C$ the total effective capacity, and $R_{1}$ the total resistance. In order to isolate the effect that it is desired to study, it is assumed that the grid of the tube is maintained at such a voltage that it absorbs no power, and that $\mu$ is a constant in the relation

$$
E_{\mathrm{p}}=\mu E_{\mathrm{g}}
$$

$E_{\mathrm{p}}=$ alternating plate voltage,

$E_{\mathrm{g}}=$ alternating voltage applied to the grid,

$\mu=$ voltage amplification factor of the tube.

That is, the tube functions as an amplifier only or that the operating point remains on the straight portion of the grid voltage, plate current curve.

2 Ballantine, Radio Telephony for Amateurs, p. 208.

B. S. Sci. Papers, No. 35 I.

1 B. S. Sci. Papers, No. 35 r, equations (2r), (22), (32), pp. 379 and 382 
Considering the circuit $L R_{1} C$

$$
E_{\mathrm{g}}=\frac{I_{1}}{j \omega C_{1}}
$$

$C_{1}=$ the capacity of the tuned circuit included between the grid and filament of the tube, $\omega=2 \pi \times$ frequency,

$$
\begin{gathered}
E_{\mathrm{p}}=\mu E_{\mathrm{g}} \\
I_{2}=\frac{E_{\mathrm{p}}}{R_{2}}=\frac{\mu I_{1}}{j \omega C_{1} R_{2}}
\end{gathered}
$$

$R_{2}=$ total resistance of the plate circuit. $^{5}$

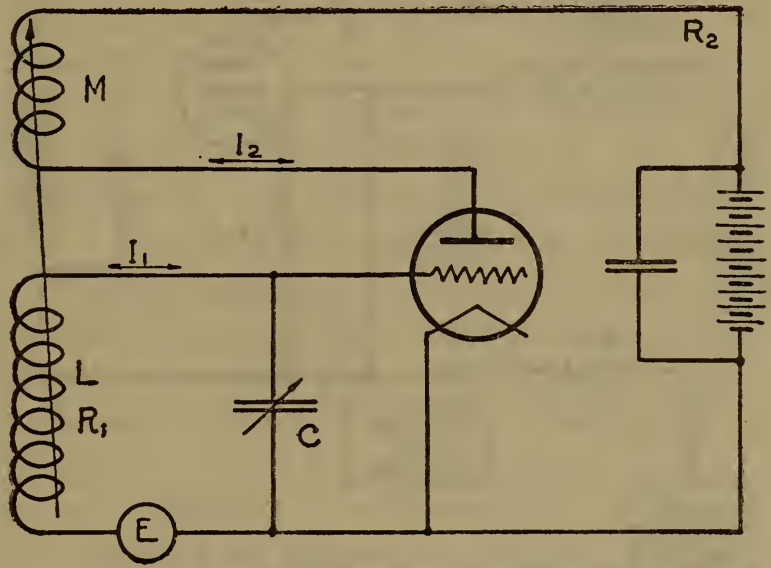

FIG. I.--Simple regenerative amplifying set.

Then the voltage $E_{M}$ fed back into the tuned circuit

$$
E_{\mathrm{M}}=i \omega M I_{2}=\frac{\mu M I_{1}}{C_{1} R_{2}}
$$

This voltage is in phase with the current in the circuit $L R_{1} C$. Then

$$
I_{1} R_{1}-\frac{\mu M I_{1}}{C_{1} R_{2}}+j I_{1} X=E
$$

where $E$ is the voltage induced by tne antenna or otner means and $X$ is reactance of circuit $L R_{1} C$.

Then

$$
I_{1}=\frac{E}{\sqrt{\left(R_{1}-\frac{\mu M}{C_{1} R_{2}}\right)^{2}+X^{2}}}
$$

The reactance of the plate circuit is neglected, as it is less than I per cent of the plate resistance of the tube.

85667-24-2 
If circuit $L R_{1} C$ is tuned to resonance with the impressed signal $E, X=O$ and

$$
I_{1}=\frac{E}{R_{1}-\frac{\mu M}{R_{2} C_{1}}}
$$

That is, the resistance is apparently reduced by an amount equal to $\frac{\mu M}{R_{2} C_{1}}$. This same result may be obtained from the equations given by Hazeltine. ${ }^{6}$

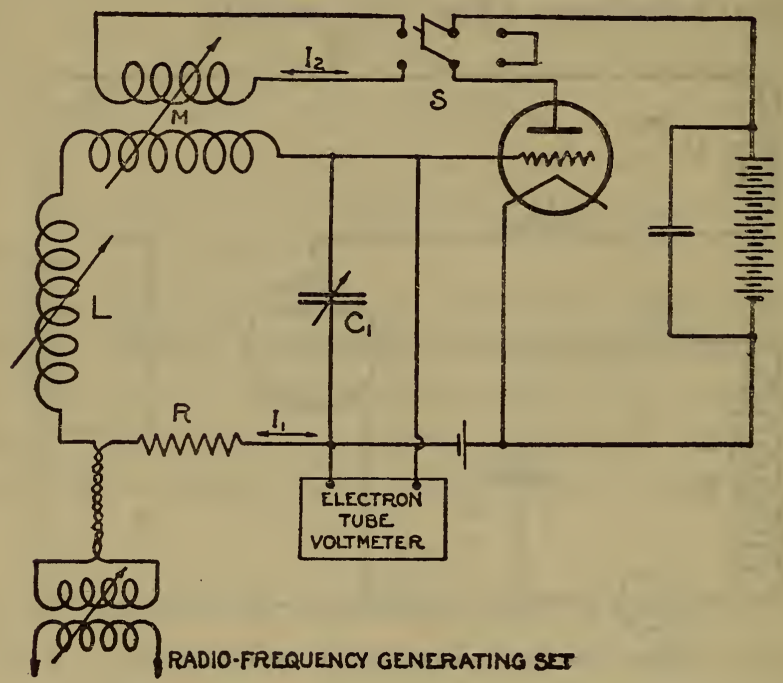

FIG. 2.-Circuit used for measurements.

3. METHOD.

In order to test this equation experimentally a circuit similar to Figure I was constructed. The actual circuit network used is shown in Figure 2. The electron-tube voltmeter measures directly the voltage impressed on the grid of the tube, and this voltage is proportional to the current flowing in the tuned circuit. The voltmeter was calibrated by measuring the voltage drop produced by a measured radio-frequency current flowing through radio-frequency link resistors of various values. There was no perceptible loss produced in the tuned circuit by connecting the tube voltmeter across the condenser. This was shown experimentally by noting the point at which self-generation set in with and without the voltmeter connected. No measurable difference 
could be detected. The capacity of the first tube of the voltmeter added to the capacity of the tuned circuit, but since it was always connected no error was introduced. The capacity $C_{1}$ between the grid and filament of the tube was kept constant. The feed back was obtained by means of varying the distance between a coil in the tuned circuit and one in the plate circuit. These coils were coaxial and separated by a grounded screen. The adjacent edges of these coils were always separated $5 \mathrm{~cm}$ or more. This mutual inductance was calibrated with all associated apparatus in place. The signal was introduced into the tuned circuit by coupling a small part of inductance $L$ to a radio-frequency generating set which was inclosed in a grounded screen box and located at considerable distance from the other apparatus.

A signal was introduced in the circuit $L R_{1} C_{1}$ and the circuit tuned to resonance with this signal. The inductor could be disconnected from the plate circuit by switch $S$. The voltage drop across the condenser $C_{1}$ was measured by means of the electrontube voltmeter. The inductor was inserted in the plate circuit, and with no coupling (coils at right angles and separated) to the tuned circuit the drop across the condenser was again measured. Then the mutual inductance was varied and the drop across the condenser measured nearly to the point of self-generation. The mutual inductance required for self-generation was noted. The tuned circuit was maintained in adjustment at all times. This procedure was repeated for different resistances in. the tuned circuit, different values of $E$, and for various frequencies.

The distributed resistance of the tuned circuit $R_{0}$ was measured by means of the resistance-variation method.

\section{RESULTS.}

From equation ( 7 ) it is evident that if $I_{0}$ is the current in the tuned circuit without regeneration and $I_{1}$ with regeneration then

$$
\frac{I_{1}}{I_{0}}=\frac{R_{1}}{R_{1}-\frac{\mu M}{C_{1} R_{2}}}
$$

that is, the amplification of the signal should be independent of the impressed voltage. Figure 3 shows the relation between $\frac{I_{1}}{I_{0}}$ and $M$ for various values of impressed voltages. This curve is continuous and the points show that $\frac{I_{1}}{I_{0}}$ is independent of $E$ for 
any value of $M$ used, the resistance remaining constant. Figure 4 shows the same relation with various values of resistance. The curves are similar and are displaced along the axis of $M$ by an amount depending on the resistance in the tuned circuit. The curves shown were taken at a frequency of 320 kilocycles per second. Curves at other frequencies are similar.

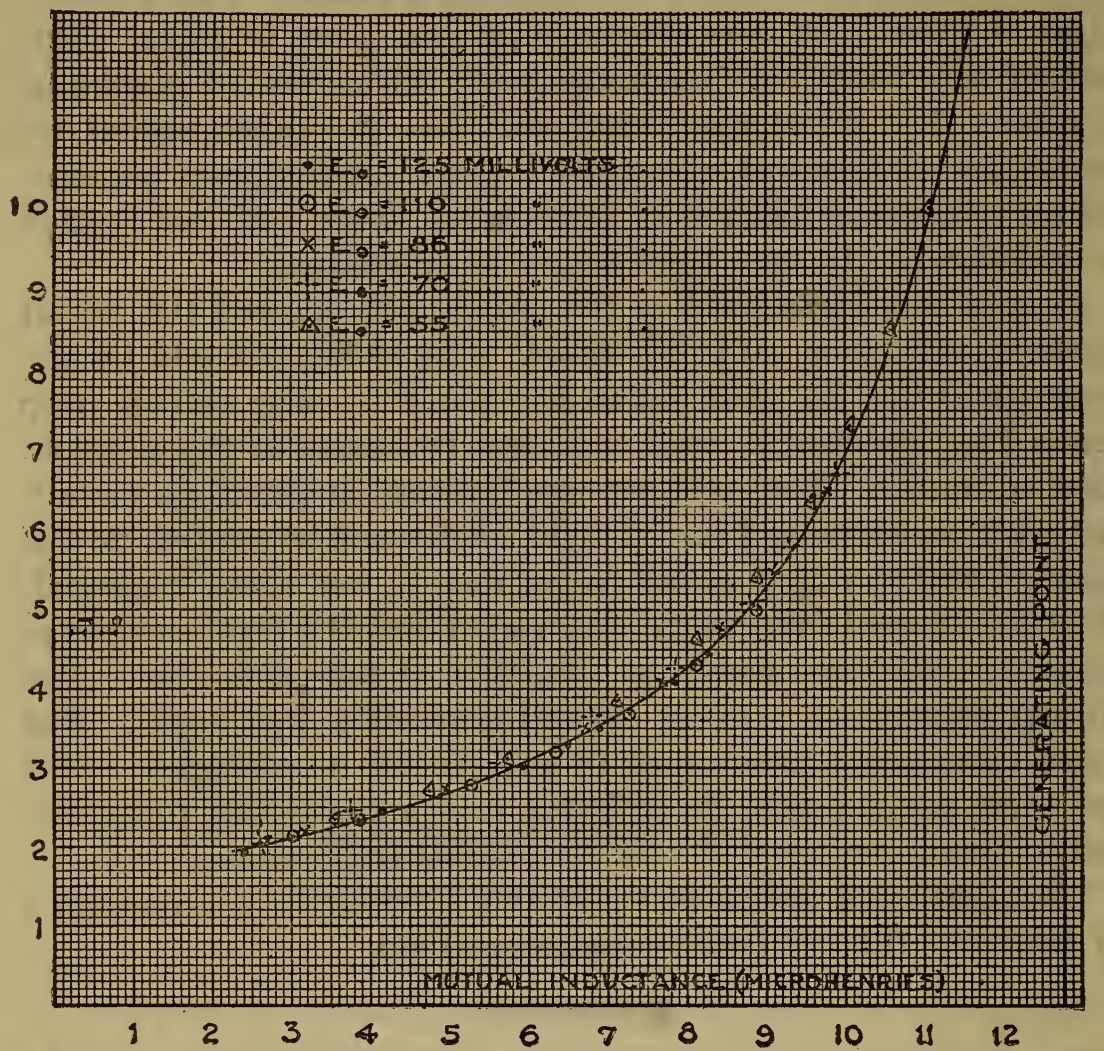

Fig. 3.-Current amplification in tuned circuit produced by regeneration for various applied voltages.

In order to test quantitatively equation (8), $\frac{I_{0}}{I_{1}}$ was plotted against $M$ for the various values of resistance. These curves should be straight lines, and from equation (8) their equation should be

$$
\frac{I_{0}}{I_{1}}=\mathrm{I}-\frac{\mu M}{R_{1} C_{1} R_{2}}
$$


The results are plotted in Figure 5 . As seen, the $\frac{I_{0}}{I_{1}}$ intercept is not unity, but the curves for various values of $R$ intersect at unity and a negative value of $M$. In plotting these curves the effect of the inductance in the plate circuit was neglected. This inductance should cause a reduction of the resistance in the

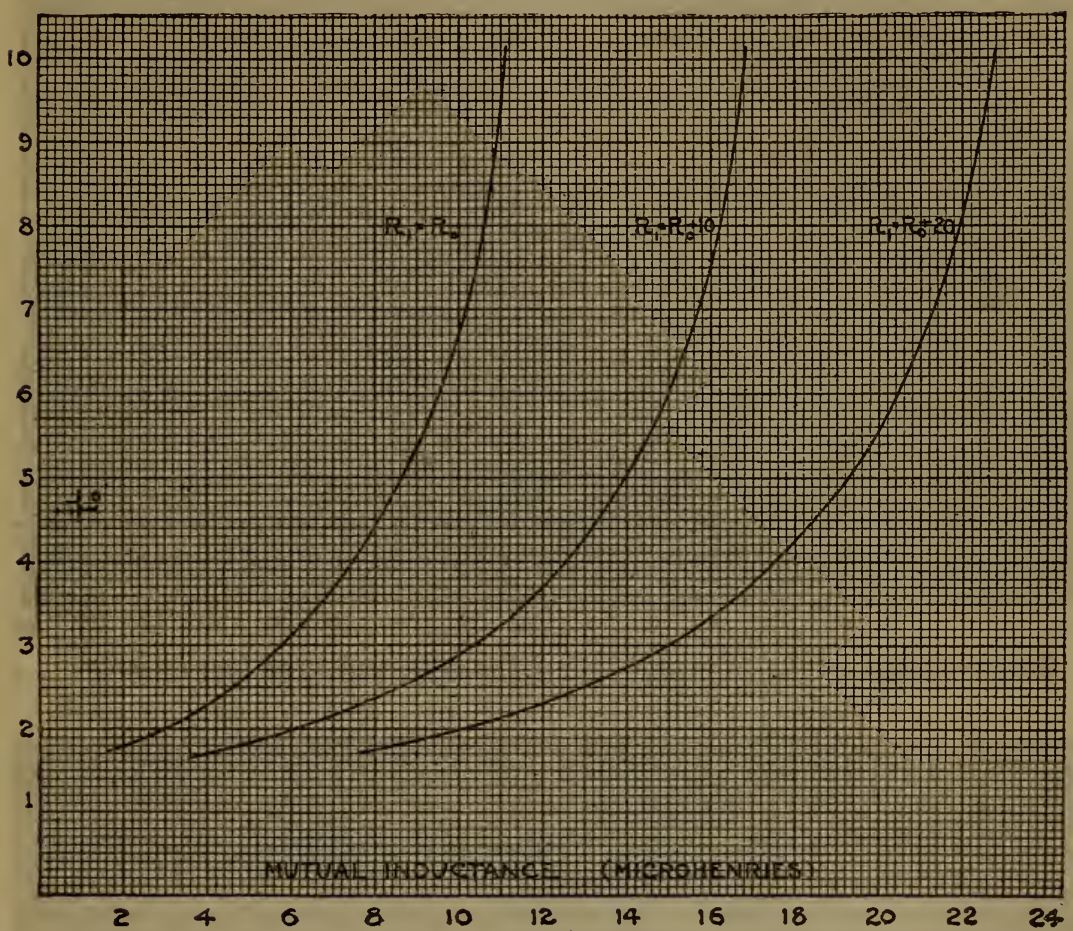

Fig. 4.-Current amplification in tuned circuit produced by regeneration for various values of resistance.

tuned circuit, the magnitude of which may be calculated from the equations of Miller.7 It was simpler, however, in this case to determine this reduction experimentally by means of the resistance-variation method. The curves for this determination are shown in Figure 6. For this case these curves show a reduction in resistance of 10.9 ohms from this cause alone.

If $a$ is this reduction of the resistance in the tuned circuit caused by the capacitive feed back through the tube the equation of the curves of Figure 5 should be

$$
\frac{I_{0}}{I_{1}}=\frac{R_{1}-a}{R_{1}}-\frac{\mu M}{R_{1} C_{1} R_{2}}
$$

${ }^{7}$ See footnote 4, p. 420. 
The intercept on the $\frac{I_{0}}{I_{1}}$ axis should be $\frac{R_{1}-a}{R_{1}}$ and the slope should be $-\frac{\mu}{R_{1} C_{1} R_{2}}$. The numerical values for the slopes and inter-

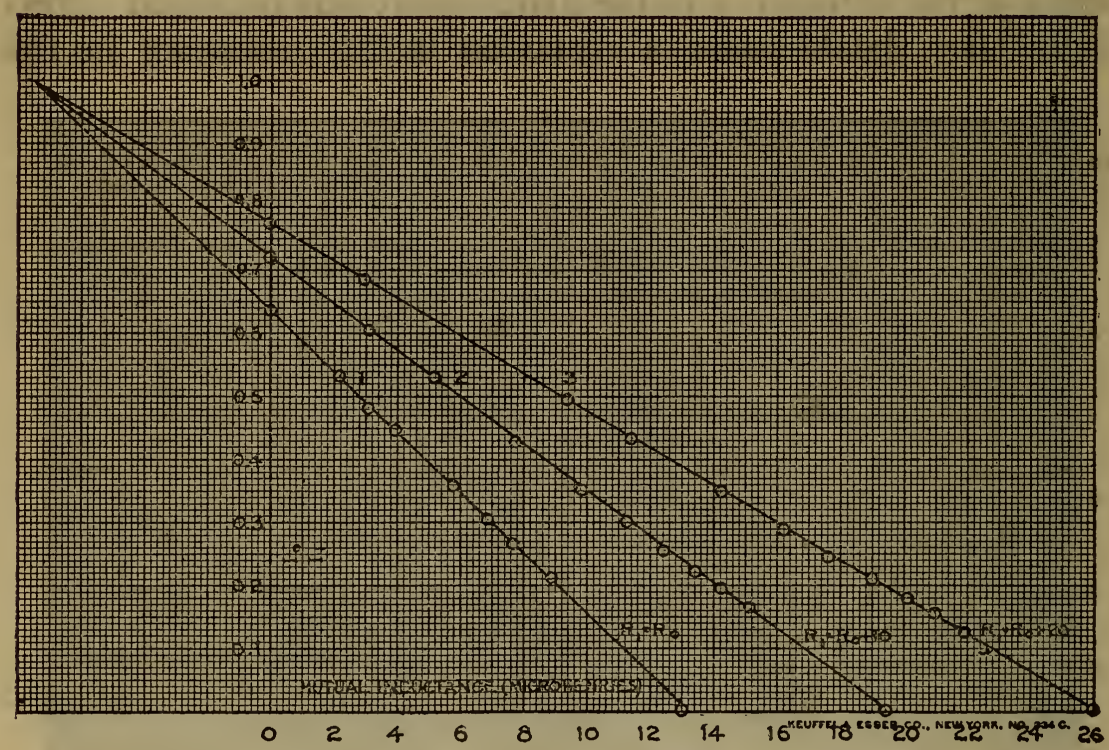

FIG. 5.-Graphical test of equation (9). Effect of capacitive feed back neglected.

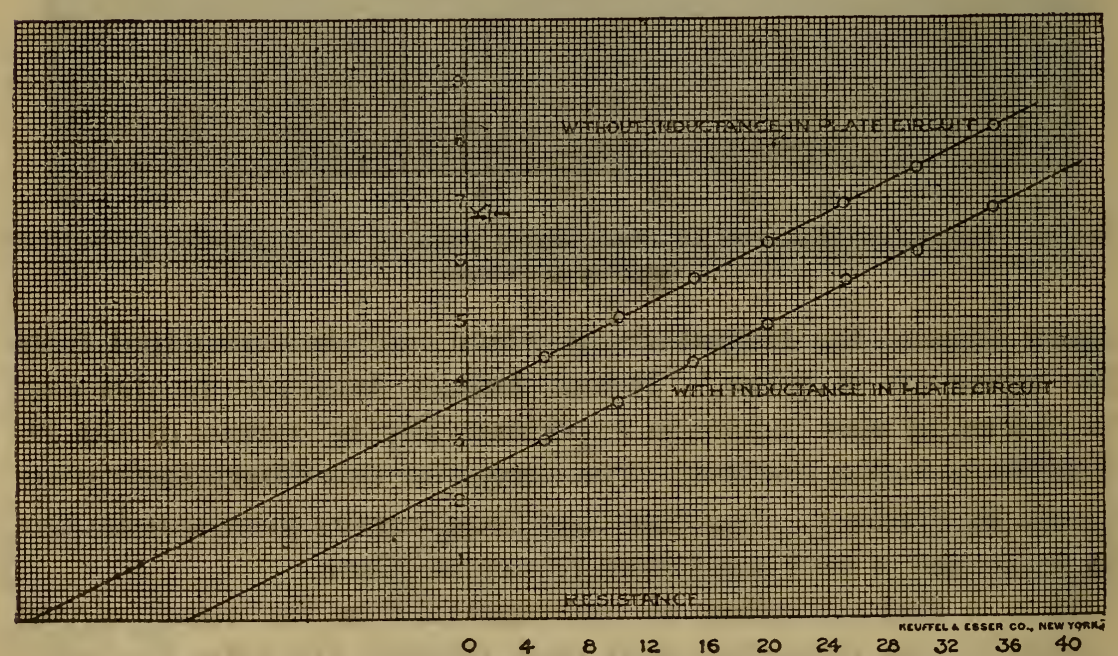

Fig. 6.-Determination of reduction of resistance caused by capacitive feed back. cepts measured from the curves of Figure 5 and the values calculated from the constants of the circuit are given in Table $I$. 
TABLE 1.-Actual and Calculated Slopes and Intercepts of Curves in Figure 5.

\begin{tabular}{|c|c|c|c|c|c|}
\hline Curve number. & $\mathbf{R}_{\mathbf{1}}$ & Intercept. & $\frac{\mathbf{R}_{1}-\mathbf{a}}{\mathbf{R}_{1}}$ & Slope. & $-\frac{\mu \mathbf{M}}{\mathbf{R}_{1} \mathbf{C}_{1} \mathbf{R}_{2}}$ \\
\hline & $\begin{array}{r}\mathbf{R}_{0} \\
\mathbf{R}_{0}+10 \\
\mathbf{R}_{0}+20\end{array}$ & $\begin{array}{l}6.25 \\
7.2 \\
7.75\end{array}$ & $\begin{array}{l}6.3 \\
7.25 \\
7.8\end{array}$ & $\begin{array}{l}-0.0480 \\
=.0368 \\
=.0297\end{array}$ & $\begin{array}{l}-0.0488 \\
=.0364 \\
=.0282\end{array}$ \\
\hline
\end{tabular}

$$
\mathrm{C}_{1}=480 \mu \mu \mathrm{l} \quad \mathbf{R}_{2}=10,400 \mathrm{ohms} \quad \mathbf{R}_{0}=29.5 \text { ohms } \quad a=10.9 \text { ohms }
$$

If instead of $I_{0}$ the value of the current $I^{\prime}{ }_{0}$ in the tuned circuit with inductance in the plate circuit and $M=O$ is used and $\frac{I_{0}^{\prime}}{I_{1}}$

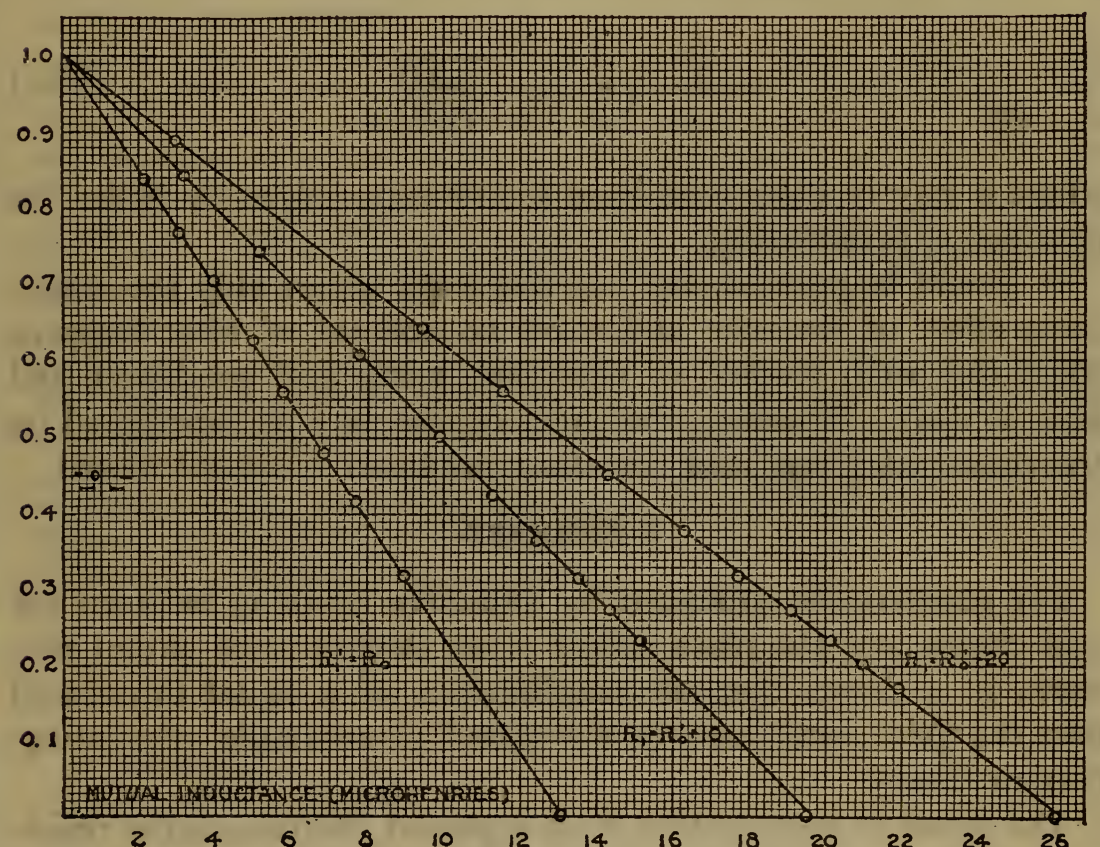

Fig. 7.-Graphical test of equation (II). Effect of capacitive feed back included.

plotted against $M$ for the same data as used for Figure 5, the equation of the curves obtained should be

$$
\frac{I^{\prime} \mathrm{o}}{I_{1}}=\mathrm{I}-\frac{\mu M}{\left(R_{1}-a\right) C_{1} R_{2}}
$$

Figure 7 shows $\frac{I^{\prime}{ }_{0}}{I_{1}}$ plotted against $M$. The intercept on the $\frac{I_{0}^{\prime}}{I_{1}}$ axis is unity in each case. Table 2 gives the numerical values of the actual and calculated slopes. 
TABLE 2.-Actual and Calculated Slopes of Curves in Figure 6.

\begin{tabular}{|c|c|c|}
\hline Curve number. & Slope. & $-\frac{\mu \mathbf{M}}{\left(\mathbf{R}_{1}-\mathbf{a}\right) \mathrm{C}_{1} \mathbf{R}_{2}}$ \\
\hline $\begin{array}{l}z_{3}^{2} \\
3\end{array}$ & $\begin{array}{l}-0.0763 \\
=.0508 \\
-.0382\end{array}$ & $\begin{array}{l}=0.0775 \\
=.00504 \\
=.0374\end{array}$ \\
\hline
\end{tabular}

These results are in good agreement with the theory and show that in order to calculate the current amplification that may be produced in a tuned circuit by regeneration by inductive feed back the following equation should be used

$$
\frac{I_{1}}{I_{0}}=\frac{R_{1}}{\left(R_{1}-a\right)-\frac{\mu M}{C_{1} R_{2}}}
$$

where $a$ may pe calculated from the formulas given by Miller ${ }^{8}$ or obtained experimentally.

It should be pointed out that this work covers only the amplifying effect of the tube and takes no account of what happens when the usual method of detection, using grid condenser and leak, is used. However, the effect of this may be superposed on the equations given, and the effect produced by it calculated.

\section{SUMMARY.}

For regeneration produced by inductive coupling between the grid circuit and the plate circuit of an electron tube circuit network, it is shown that the amplification of a signal introduced into the grid circuit can be calculated from simple alternatingcurrent theory.

The result of regeneration is an apparent decrease in the resistance of the tuned circuit. The equations derived are verified experimentally.

WASHINGTON, January 2, 1924.

${ }^{8}$ See footnote 4 , p. 420 . 